\title{
CD4 Positive Memory T-Lymphocyte
}

National Cancer Institute

\section{Source}

National Cancer Institute. CD4 Positive Memory T-Lymphocyte. NCI Thesaurus. Code C97349.

A mature $T$ lymphocyte that has encountered antigen at a previous time and is primed to react strongly during its next encounter with that antigen. These cells can be identified by the presence of both the CD4 and CD45RO molecules on their surface. 